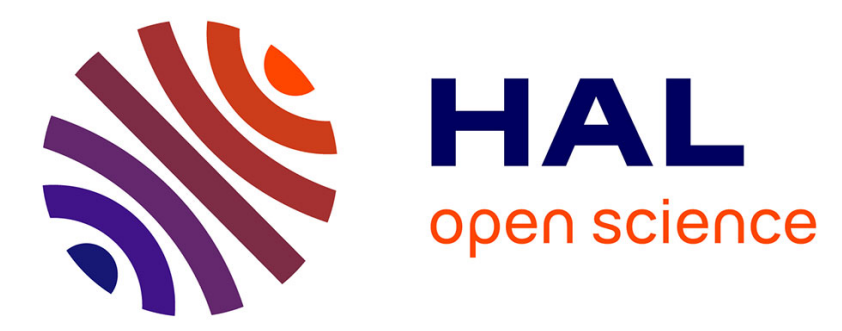

\title{
Finite-time observer-based backstepping control of a flexible launch vehicle
}

\author{
Elodie Duraffourg, Laurent Burlion, Tarek Ahmed-Ali
}

\section{To cite this version:}

Elodie Duraffourg, Laurent Burlion, Tarek Ahmed-Ali. Finite-time observer-based backstepping control of a flexible launch vehicle. Journal of Vibration and Control, 2016, 24 (8), pp.1535-1550. 10.1177/1077546316664021. hal-02315092

\section{HAL Id: hal-02315092 \\ https://hal.science/hal-02315092}

Submitted on 2 Nov 2021

HAL is a multi-disciplinary open access archive for the deposit and dissemination of scientific research documents, whether they are published or not. The documents may come from teaching and research institutions in France or abroad, or from public or private research centers.
L'archive ouverte pluridisciplinaire HAL, est destinée au dépôt et à la diffusion de documents scientifiques de niveau recherche, publiés ou non, émanant des établissements d'enseignement et de recherche français ou étrangers, des laboratoires publics ou privés.

\section{(c)(1)}

Distributed under a Creative Commons Attribution| 4.0 International License 


\title{
Finite-time observer-based backstepping control of a flexible launch vehicle
}

\author{
Elodie Duraffourg', Laurent Burlion' and Tarek Ahmed-Ali ${ }^{2}$
}

\begin{abstract}
In this paper, a longitudinal model of a space launch vehicle was developed using the Lagrange mechanism and a free-free Euler-Bernoulli beam model. The aim was to propose a model including one flexible mode plus a nonlinear aerodynamic coefficient for nonlinear control design. We then studied the output feedback problem raised by using such a nonlinear model. The main achievement is to propose a new finite-time state observer when the measured outputs are corrupted by an unidentified flexible mode. This effect may destabilize a classical backstepping control law applied to the rigid model. To achieve this, a backstepping control law was redesigned to damp out the flexible mode, once measured and characterized. Hence a new adaptive finite time observer was developed. Closed-loop simulations show the effectiveness of the observer in combination with a redesigned backstepping control law when sensors and the launcher nozzle are collocated.
\end{abstract}

Keywords

Nonlinear control, backstepping, observer design, flexible modes

\section{Introduction}

The problem of finite-time observation for linear and nonlinear systems has been widely investigated over the past decades. Two classes of observers emerge in the series of methods that achieve finite-time convergence. The first one, based on the use of delay, has deserved a lot of attention (Engel and Kreisselmeier, 2002; Menold et al., 2003). Recently, in Karafyllis and Jiang (2011), a novel hybrid dead-beat observer which uses delays has been proposed. The history of the output is used in order to estimate the state of the system. Sliding mode observers, widely researched in the literature, make up the second class (see AhmedAli and Lamnabhi Lagarrigue, 1999; Shtessel et al., 2010, for instance). More recently, homogeneous finite-time observers have been developed for a specific class of nonlinear system in Perruquetti et al. (2008). Most of these approaches make the assumption that the system structure and parameters are known.

In this paper we propose to design a finite-time observer for a space launch vehicle which belongs to the class of uncertain nonlinear systems. Researchers have recently investigated this subject in the field of nonlinear control. Several solutions have been proposed. Some of them, on the one hand, addressed the problem of unknown parameters and uncertainties using a direct-adaptive, (Fiorentini et al., 2009; Hu et al., 2012), or time varying controller (Hervas and Reyhanoglu, 2012). Nevertheless, only rigid states (attitude angle and pitch rate) are used in these proposed methods. A great deal of further effort has been devoted to stabilize uncertain nonlinear systems with disturbances (Pan et al., 2015a; Yu et al., 2015). Such control methods are also applied to vehicle suspension systems (Pan et al., 2015b; Sun et al., 2016). Current active research in the field of flexible robots also aims at achieving robustness to model uncertainties and disturbances (see, e.g., De Luca, 2015, and references therein).

On the other hand, estimating the flexible states is often necessary for control design purposes: for instance, $\mathrm{Hu}$ (2009) uses them to actively reduce the vibrations of a flexible spacecraft. Using a sliding

\footnotetext{
'Onera, the French Aerospace Lab, France

${ }^{2}$ Ecole Nationale Supérieure d'Ingénieurs de Caen (ENSICAEN), France Received: 27 November 2015; accepted: 18 July 2016

Corresponding author:

Laurent Burlion, The French Aerospace Lab, F-31055 Toulouse, France. Email: Iburlion@onera.fr
} 
mode state observer, Shtessel et al. (2010) estimate the flexible states (mode shape coordinates and their time derivatives) in order to remove the undesirable dynamics from the measurements. These approaches unfortunately require a strong knowledge of the mathematical model of the system, in particular parameters for the flexible modes (natural frequency and damping of the bending modes). However, to the best of our knowledge, the design of a finite-time observer has not been achieved on uncertain nonlinear aerospace models.

Due to mass constraints, space vehicles tend to have lightweight and flexible structures with low natural frequencies, distorting sensor measurements and adding stability problems during flight. Indeed, sensor measurements deliver rigid launcher motion plus flexible displacements at the location of the sensor. Consequently, the location of the sensors has a significant importance (Frosch and Vallely, 1967). Notch-filters are usually used to overcome this stability problem by filtering the bending mode and reverting to rigid body behavior. However, this no longer works if the flexible natural frequency is very low because of interaction with rigid dynamics. Another proposed solution consists in estimating the flexible states to filter the outputs.

As far as we are concerned, we recently designed a Lyapunov-based nonlinear controller, which uses the flexible states, to ensure control objectives of both reference path tracking and bending mode damping for a class of nonlinear flexible systems (Burlion et al., 2013); (Duraffourg et al., 2013a); (Duraffourg et al., 2013b). Assuming that the whole state is available, this control law has been applied to the rotational dynamics of a space launch vehicle in Duraffourg et al. (2013c). Such assumptions do not hold in practical applications since flexible states are generally not measured. Consequently, we need to estimate the flexible states. Besides, noting that flexible parameters are subject to uncertainties or variation during flight, this paper aims to extend existing theory by proposing an indirect adaptive hybrid observer that no longer requires system parameter knowledge. The proposed approach, which extends the preliminary conference paper of Duraffourg et al. (2014) consists of estimating flexible parameters (natural frequency and damping) and initial state conditions, using the algebraic tools of Fliess and Sira-Ramirez (2003). The first ones improve the accuracy and the robustness of the observer through the indirect adaptive feature. The second ones are used to regularly update the estimated state and so guarantee a finite-time convergence.

This paper is organized as follows. Section 2 formulates the mathematical model of the flexible launch vehicle using the Lagrange mechanism and a free-free Euler-Bernoulli beam model. In Section 3, the control problem is stated. A nonlinear control law, using the unmeasured flexible states, and achieving control objectives of stability and bending mode damping, is presented. Since flexible states are required, Section 4 develops a flexible parameter estimator and a flexible state observer which are then mixed to design a hybrid adaptive finite-time observer. Simulation results are given in Section 5. In section 6, the blending of the finite-time observer and nonlinear control law is discussed in addition to the closed-loop simulations. Closed-loop stability properties are also analyzed. Finally, Section 7 contains our conclusions and future research directions.

\section{I.I. Useful notation}

Useful notation is reported in Table 1 .

Table I. Nomenclature.

\begin{tabular}{|c|c|c|}
\hline Notation & Meaning & Unit \\
\hline & $\begin{array}{l}\text { Deviation angle around axis w.r.t } \\
\text { the guidance attitude refer- } \\
\text { ence angle }\end{array}$ & $\mathrm{rad}$ \\
\hline$\beta$ & Thruster angle of deflection & $\mathrm{rad}$ \\
\hline$\eta$ & $\begin{array}{l}\text { Mode shape temporal } \\
\text { coordinate }\end{array}$ & \\
\hline$q$ & Pitch rate & $\mathrm{rad} / \mathrm{s}$ \\
\hline y & Drift & $\mathrm{m}$ \\
\hline$h_{\mathrm{T}}$ & Thruster flexible displacement & $\mathrm{m}$ \\
\hline$r_{\mathrm{T}}$ & Thruster flexible rotation & $\mathrm{rad}$ \\
\hline$r_{\mathrm{ci}}$ & Inertial unit flexible rotation & $\mathrm{rad}$ \\
\hline$r_{g y}$ & Rategyro flexible rotation & $\mathrm{rad}$ \\
\hline$T$ & Thrust & $\mathrm{kg} \mathrm{m} / \mathrm{s}^{2}$ \\
\hline$L$ & Lift & $\mathrm{kg} \mathrm{m} / \mathrm{s}^{2}$ \\
\hline$D$ & Drag & $\mathrm{kg} \mathrm{m} / \mathrm{s}^{2}$ \\
\hline$G_{L}$ & Launcher center of mass & \\
\hline$C_{T}$ & Gimbal joint & \\
\hline$F_{L}$ & Aerodynamic center & \\
\hline$I_{L}$ & Launcher body inertia & $\mathrm{kg} \mathrm{m}^{2}$ \\
\hline$M_{L}$ & Launcher body mass & $\mathrm{kg}$ \\
\hline$L_{T}$ & Algebraic distance from $G_{L}$ to $C_{T}$ & $\mathrm{~m}$ \\
\hline$I_{\text {aero }}$ & Algebraic distance from $F_{L}$ to $G_{L}$ & $\mathrm{~m}$ \\
\hline $\bar{q}$ & Dynamic pressure & $\mathrm{Pa}$ \\
\hline$S$ & Reference area of the vehicle & $\mathrm{m}^{2}$ \\
\hline$\omega$ & $\begin{array}{l}\text { Natural frequency of the first } \\
\text { bending mode }\end{array}$ & $\mathrm{rad} / \mathrm{s}$ \\
\hline $\mathcal{R}_{n}$ & $\begin{array}{l}\text { Frame }\left(G_{n}, x_{n}, y_{n}\right) \text { linked to the } \\
\quad \text { reference trajectory }\end{array}$ & \\
\hline$\xi$ & $\begin{array}{l}\text { Natural damping of the first } \\
\text { bending mode }\end{array}$ & \\
\hline $\mathcal{R}_{L}$ & $\begin{array}{l}\text { Frame }\left(G_{L}, x_{L}, y_{L}\right) \text { linked to the } \\
\text { launcher }\end{array}$ & \\
\hline
\end{tabular}




\section{Modeling of a flexible launch vehicle}

This section states the longitudinal dynamics of a launch vehicle during its atmospheric flight.

\section{I. Launch vehicle}

We consider a flexible, static (masses and inertia stay constant) and symmetric space vehicle moving around its center of mass $G_{L}$, as shown in Figure 1. The launcher is propelled thanks to a thrust force $T$ oriented by a deflection angle $\beta$. The launcher dynamics is formulated in the fixed and non-Galilean frame $R_{n}=\left(G_{n}, x_{n}, y_{n}\right)$ linked to the reference trajectory. Let us note $R_{L}=\left(G_{L}, x_{L}, y_{L}\right)$ the frame attached to the launcher body, $\psi$ the deviation angle around the body axis with respect to the guidance attitude reference and $y$ the position along the $y_{n}$ axis.

To capture flexible effects, we assume that the launcher body can be identified with a free-free EulerBernoulli beam model on which the separation principle has been applied. Solving the Euler-Lagrange equation gives a general solution (in the free vibration case) which depends on the so-called displacements solutions. It is assumed that the control synthesis can be achieved considering merely the lower frequency displacement solution (also called the first bending mode), the remaining flexible modes being ignored. Indeed, it is well known that this assumption is satisfactory if the closed-loop bandwidth is below a critical limit. The launcher flexible displacements along the lateral axis $y_{L}$ caused by the first bending mode is given by

$$
u(x, t)=h(x) \eta(t)
$$

where $h(x)$ is the first mode shape and $\eta(t)$ is the temporal mode shape coordinate. Let us also define the mode shape derivative with respect to the launcher axis coordinate (that corresponds to a flexible rotation) by

$$
r(x)=\frac{d h(x)}{d x}
$$

Figure 2 illustrates flexible displacements at a given instant $t$, along the launcher axis for the first bending mode and gives a schematic representation of the mode shape. The following notations are considered

$$
\begin{aligned}
& r_{T}=r\left(x_{T}\right) \quad h_{T}=h\left(x_{T}\right) \quad r_{c i}=r\left(x_{c i}\right) \\
& r_{g y}^{a}=r\left(x_{g y}^{a}\right) \quad r_{g y}^{b}=r\left(x_{g y}^{b}\right)
\end{aligned}
$$

where $x_{T}, x_{\mathrm{ci}}, x_{g y}^{a}$ and $x_{g y}^{b}$ respectively are the coordinates of the gimbal joint, inertial unit, rate-gyro " $a$ " and rate-gyro " $b$ " on the launcher body axis $x_{L}$.

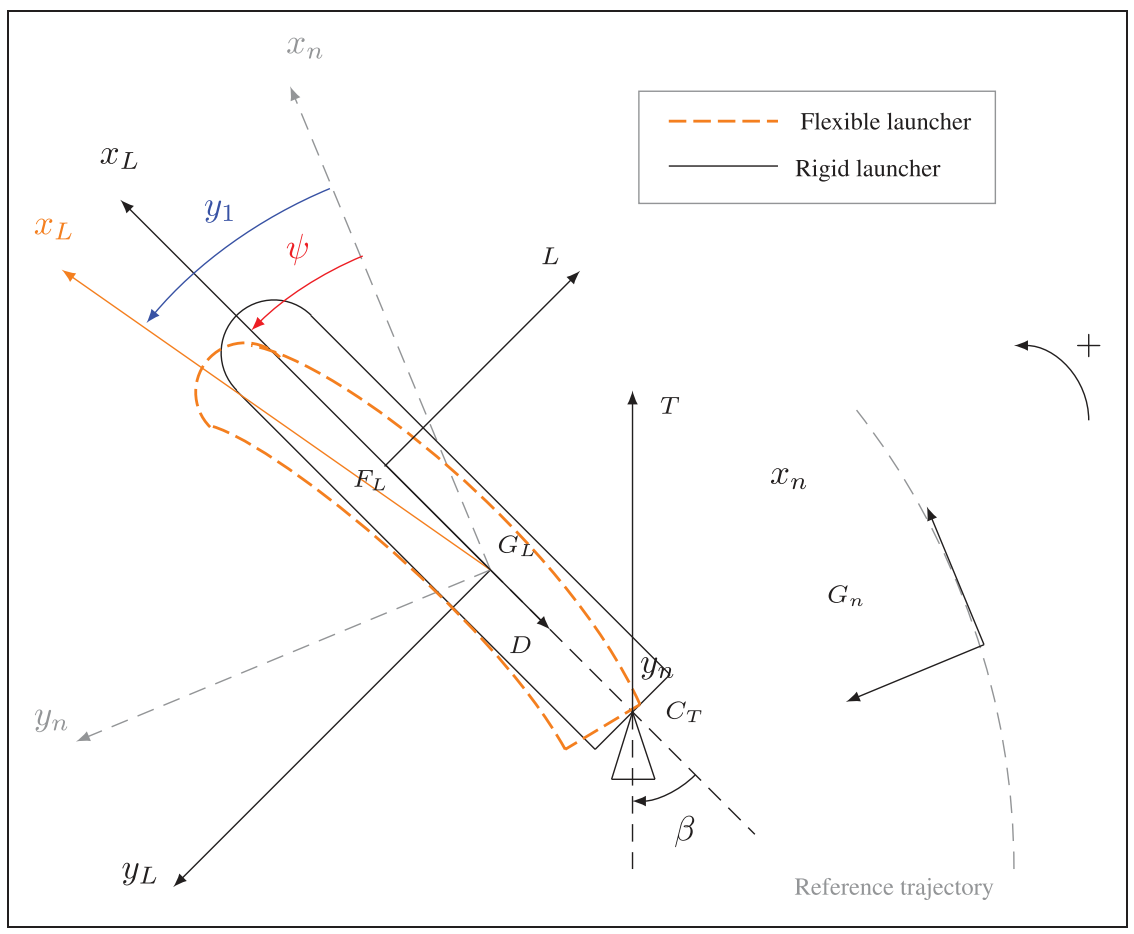

Figure I. Flexible launch vehicle schematic representation. 


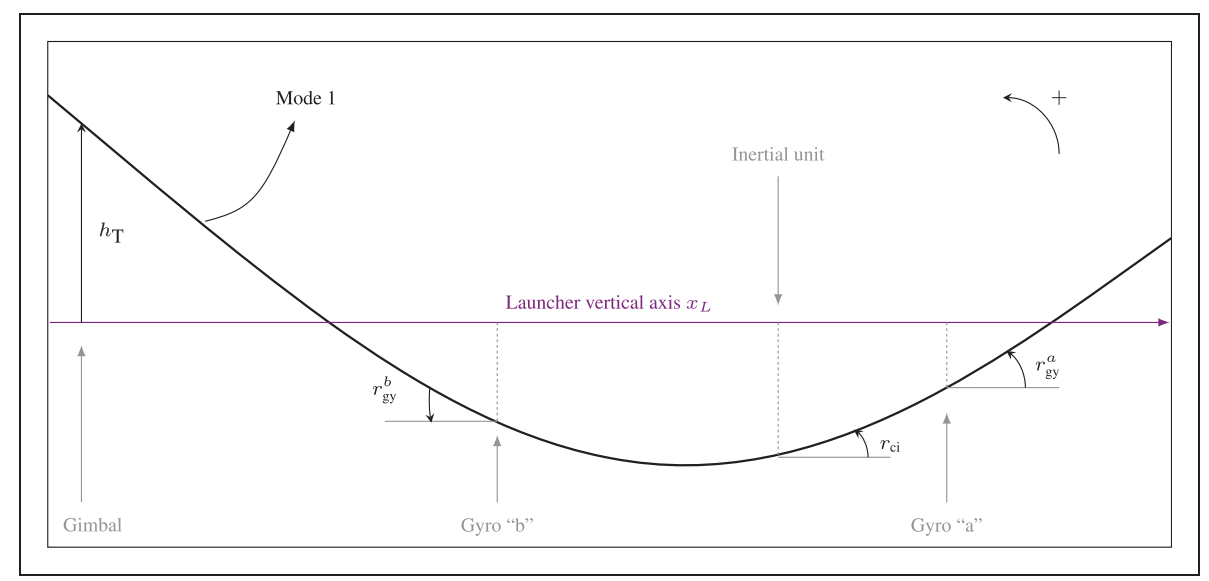

Figure 2. Schematic view of the first mode shape.

\subsection{Mathematical model}

The launcher mathematical model is extracted from the Lagrangian mechanism. As in Clement (2001) and Clement et al. (2001), this model is a deviation model with respect to a reference model. The Lagrangian generalized coordinates are referred to as $\kappa=(y, \psi, \eta)$ and the Lagrangian takes the form $\mathcal{L}=\mathcal{L}(\kappa, \dot{\kappa})$. Dynamic equations of the flexible launch vehicle are given by

$$
\begin{gathered}
\frac{d}{d t} \frac{\partial \mathcal{L}}{\partial \dot{y}}-\frac{\partial \mathcal{L}}{\partial y}=\Phi_{y} \\
\frac{d}{d t} \frac{\partial \mathcal{L}}{\partial \dot{\psi}}-\frac{\partial \mathcal{L}}{\partial \psi}=\Phi \\
\frac{d}{d t} \frac{\partial \mathcal{L}}{\partial \dot{\eta}}-\frac{\partial \mathcal{L}}{\partial \eta}+\frac{\partial \mathcal{R}}{\partial \dot{\eta}}=\Phi_{\eta}
\end{gathered}
$$

where $\mathcal{R}=\xi \omega \dot{\eta}^{2}$ is a Rayleigh dissipation function introduced to consider natural energy dissipation of the bending mode and $\Phi_{j}$ is the generalized force relative to Lagrangian coordinate $j \in\{y, \psi, \eta\}$.

Aerodynamic forces (lift $L$ and drag $D$ ), thrust $T$, and inertial forces (since the fixed frame is not Galilean) make up the external forces applied to the launch vehicle. Their generalized forces are formulated as follows

$$
\begin{aligned}
& \Phi_{y}=D \sin \psi+L \cos \psi+T \sin \left(\psi+\beta+r_{\mathrm{T}} \eta\right) \\
& \Phi_{\psi}=-L l_{\text {aero }}+T L_{\mathrm{T}} \sin (\beta+r \eta)-T h_{\mathrm{T}} \eta \cos \left(\beta+r_{\mathrm{T}} \eta\right) \\
& \Phi_{\eta}=h_{\mathrm{T}} T \sin \left(\beta+r_{\mathrm{T}} \eta\right)
\end{aligned}
$$

We compute kinetic and potential energies of the launcher in order to apply the energetic formulation of the Lagrange mechanism. Kinetic energies of the launch vehicle $\mathcal{T}_{L}$ and the shape mode $\mathcal{T}_{f}$ are expressed as follows

$$
\begin{gathered}
\mathcal{T}_{L}=\frac{1}{2} M_{L}\left(\dot{x}^{2}+\dot{y}^{2}\right)+\frac{1}{2} I_{L} \dot{\psi}^{2} \\
\mathcal{T}_{f}=\frac{1}{2} \dot{\eta}^{2}
\end{gathered}
$$

The bending mode is identified as a mass-spring system whose stiffness is linked to its natural frequency $k=\omega^{2}$. Thus, the elastic potential energy is

$$
\mathcal{V}_{f}=\frac{1}{2} \omega^{2} \eta^{2}
$$

Note that $V_{f}$ is the total potential energy of the deviation model with respect to the reference model, which takes into account the potential energy due to gravity.

Then, the Lagrangian is stated as the difference between the kinetic and the potential energies. That is

$$
\mathcal{L}=\mathcal{T}_{L}+\mathcal{T}_{f}-\mathcal{V}_{f}
$$

Applying Lagrangian formalism (4)-(6) with the generalized forces (7), the equations of motion of the launch vehicle are obtained

$$
\left\{\begin{array}{l}
M_{L} \ddot{y}=D \sin \psi+L \cos \psi+T \sin \left(\psi+\beta+r_{\mathrm{T}} \eta\right) \\
I_{L} \ddot{\psi}=-L l_{\text {aero }}+T L_{\mathrm{T}} \sin \left(\beta+r_{\mathrm{T}} \eta\right) \\
\quad-T h_{\mathrm{T}} \eta \cos \left(\beta+r_{\mathrm{T}} \eta\right) \\
\ddot{\eta}=-\omega^{2} \eta-2 \xi \omega \dot{\eta}+h_{\mathrm{T}} T \sin \left(\beta+r_{\mathrm{T}} \eta\right)
\end{array}\right.
$$

The spacecraft is equipped with two rate-gyros and an inertial unit that delivers measurements corrupted by flexible mode shape, as shown in Figure 1, 
where $y_{1}$ does not exactly match with

$$
\left\{\begin{array}{l}
y_{1}=\psi+r_{\mathrm{ci}} \eta \\
y_{2}=q+r_{g y} \dot{\eta}
\end{array}\right.
$$

where $r_{\mathrm{gy}}$ is equal to $r_{g y}^{a}$ or $r_{g y}^{b}$ depending on whether rate-gyro " $a$ " or " $b$ " is considered. These two separate cases will be studied in Section 7.

\section{Problem statement}

Due to the classical time-scale separation principle, throughout the remainder of the paper, we focus only on fast dynamics (that is, rotational dynamics), and make the assumption of small angles for the actuator and flexible rotations. In other words, we only consider the two last equations of (11) where the trigonometric functions have been linearized. The control input is the thruster angle of deflection $\beta$. Using the notation $q$ for the pitch rate, the considered equations of motion are given by

$$
\left\{\begin{array}{l}
\dot{\psi}=q \\
\dot{q}=-\frac{l_{\text {aero }}}{I_{L}} L(\psi)+\mathbf{C}_{\mathbf{N}} \mathbf{N}+d_{N} \beta \\
\dot{\mathbf{N}}=\mathbf{A}_{\mathbf{N}} \mathbf{N}+\mathbf{B}_{\mathbf{N}} \beta
\end{array}\right.
$$

with $\mathbf{N}=[\eta, \dot{\eta}]^{\mathrm{T}}$ and

$$
\begin{aligned}
& \mathbf{A}_{\mathbf{N}}=\left(\begin{array}{cc}
01 \\
-\omega^{2}+h_{\mathrm{T}} r_{\mathrm{T}} T-2 \xi \omega
\end{array}\right) ; \quad \mathbf{B}_{\mathbf{N}}=\left(\begin{array}{c}
0 \\
h_{\mathrm{T}} T
\end{array}\right) \\
& \mathbf{C}_{\mathbf{N}}=\left(\begin{array}{ll}
\frac{T}{I_{L}}\left(L_{\mathrm{T}} r_{\mathrm{T}}-h_{\mathrm{T}}\right) & 0
\end{array}\right) ; \quad d_{N}=\frac{T L_{T}}{I_{L}}
\end{aligned}
$$

In the remainder of the paper, $(\psi, q)$ are referred to as rigid states while $(\eta, \dot{\eta})$ are referred to as flexible states. As stated in Fiorentini et al. (2009), for a hypersonic aircraft lift $L(\psi)$ is classically described by a polynomial function (see also Boiffier (1998) for more details on aerodynamics coefficients)

$$
L(\psi)=\bar{q} S\left(C_{L}^{1} \psi-C_{L}^{2} \psi^{2}\right)
$$

Consider system (13). Our objective is twofold:

- to steer the attitude angle to zero so that the vehicle keeps the guidance reference trajectory;

- to add damping to the bending mode.

The challenge consists in considering this problem when:

- the model possesses a polynomial nonlinearity (here $L(\psi))$;

- the natural damping and pulsation of the flexible mode are unknown;
- we do not measure the full state of the system (we only measure (12).

We address this problem under the following assumptions:

Assumption 1. All parameters are supposed to be known, except $\xi$ and $\omega$ which are only bounded.

Remark 1. The parameters which account for flexible dynamics are usually known with a higher level of uncertainty than those which account for rigid dynamics. Therefore, the project focused on studying the uncertainties in damping ratio $\xi$ and the natural frequency $\omega$ of the flexible mode. To be more thorough, we should also consider uncertainties of $r_{T}, h_{\mathrm{T}}, r_{c i}$ and $r_{g i}$. This would certainly greatly complicate the proposed observer synthesis and is postponed to future studies.

Remark 2. An additional persistence of excitation (PE) assumption, detailed in Section 5, boils down to the fact that the output signals are sufficiently "rich" to estimate the unknown flexible parameters. See Subsection 6.3.2 for more details.

A nonlinear control law which achieves the control objectives has been developed in Duraffourg et al. (2013a). This controller is referred to as "flexible backstepping," since it uses the flexible states and minimizes the impact of the rigid dynamics on the transience of the flexible one. Despite this, this control law consists in a full-state feedback and requires knowing flexible states and parameters. That's why we here propose to build a dedicated adaptive observer in order to estimate the flexible states together with the unknown parameters.

\section{I. Nonlinear control law design for the rotational dynamics}

Before giving the observer synthesis, we firstly show how one can build a state-feedback nonlinear controller which achieves the control objectives when the flexible parameters are known.

Let us note

$$
c_{\beta}=\frac{I_{L} h_{T}}{L_{T}}
$$

We firstly rewrite system (13), applying the following change of coordinates to the flexible dynamics

$$
\mathbf{z}=\left(\begin{array}{c}
\eta \\
\dot{\eta}
\end{array}\right)-c_{\beta}\left(\begin{array}{l}
q \\
q
\end{array}\right)
$$


It yields

$$
\begin{aligned}
& \dot{\psi}=q \\
& \dot{q}=-\frac{l_{\text {aero }}}{I_{L}} L(\psi)+\mathbf{C}_{\mathbf{N}} \mathbf{N}+d_{N} \beta \\
& \dot{\mathbf{z}}=\mathbf{A}_{\mathbf{z}} \mathbf{z}+\mathbf{F}(\psi) \psi+\mathbf{G} q
\end{aligned}
$$

with

$$
\begin{aligned}
\mathbf{A}_{\mathbf{z}} & =\mathbf{A}_{\mathbf{N}}-c_{\beta}\left(\begin{array}{ll}
0 & 0 \\
\mathbf{C}_{\mathbf{N}}
\end{array}\right) \\
\mathbf{F}(\psi) \psi & =c_{\beta}\left(\mathbf{A}_{\mathbf{N}}\left(\begin{array}{l} 
\\
0
\end{array}\right)+\frac{l_{\text {aero }}}{I_{L}}\left(\begin{array}{c}
0 \\
L(\psi)
\end{array}\right)\right) \\
\mathbf{G} & =c_{\beta}\left(\mathbf{A}_{\mathbf{N}}\left(\begin{array}{l}
0 \\
1
\end{array}\right)-\left(\begin{array}{l}
1 \\
0
\end{array}\right)\right)
\end{aligned}
$$

The remaining q-term inżsuggests the following second change of coordinates

$$
\mathbf{Z}=\mathbf{z}-\mathbf{G} \psi
$$

We obtain a nonlinear system in lower triangular form

$$
\left\{\begin{array}{l}
\dot{\mathbf{Z}}=\mathbf{A}_{\mathbf{z}} \mathbf{Z}+\mathbf{H}(\psi) \psi \\
\dot{\psi}=q \\
\dot{q}=-\frac{l_{\text {eero }}}{I_{L}} L(\psi)+\mathbf{C}_{\mathbf{N}} \mathbf{N}+d_{N} \beta
\end{array}\right.
$$

where $\mathbf{H}(\psi):=\mathbf{F}(\psi)+\mathbf{A}_{\mathbf{z}} \mathbf{G}$

Proposition 1. Consider system (19) with the following feedback control law

$$
\begin{aligned}
\beta_{\mathrm{f}}(\psi, q, \mathbf{N})= & \frac{c_{\beta}}{h_{\mathrm{T}} T}\left[\dot{q}_{\mathrm{cmd}}+\frac{l_{\text {aero }}}{I_{L}} L(\psi)\right. \\
& \left.-\mathbf{C}_{\mathbf{N}} \mathbf{N}-\frac{1}{c_{3}^{\mathrm{f}}}\left(c_{1}^{\mathrm{f}} \psi+\lambda_{q} \delta_{q}\right)\right]
\end{aligned}
$$

with $\lambda_{q}>0, \lambda_{\psi}>0$ and where

$$
q_{\mathrm{cmd}}=-\frac{1}{c_{1}^{\mathrm{f}}}\left(\lambda_{\psi} \psi+c_{2}^{\mathrm{f}} \mathbf{H}(\psi)^{\mathrm{T}} \mathbf{P}_{\mathbf{z}} \mathbf{Z}\right) ; \quad \delta_{q}=q-q_{\mathrm{cmd}}
$$

control parameters $c_{i}^{\mathrm{f}} \quad i \in\{1,2,3\}$ are positive (tuning) constants and $\mathbf{P}_{\mathbf{z}} \in \mathbb{R}^{2 \times 2}$ is a symmetric positive definite matrix defined hereafter.

So, the closed-loop system is globally asymptotically stable at the origin. This proposed controller also leads to global asymptotic stability (GAS) of the closed-loop system composed by (13) and (20).

Proof. $\mathbf{A}_{\mathbf{z}}$ being Hurwitz, flexible backstepping control law can be applied to system (17) (see Duraffourg et al., 2013a,b), considering the following Lyapunov function

$$
V_{f}=\frac{c_{1}^{\mathrm{f}}}{2}{ }^{2}+\frac{c_{2}^{\mathrm{f}}}{2} \mathbf{Z}^{\mathrm{T}} \mathbf{P}_{\mathbf{z}} \mathbf{Z}+\frac{c_{3}^{\mathrm{f}}}{2} \delta_{q}^{2}
$$

where $c_{i}^{\mathrm{f}}$ are positive constants $(i \in\{1,2,3\}), \boldsymbol{P}_{z} \in \mathbb{R}^{2 \times 2}$ is the positive and symmetric matrix which verifies

$$
\mathbf{A}_{\mathbf{z}}{ }^{\mathrm{T}} \mathbf{P}_{\mathbf{z}}+\mathbf{P}_{\mathbf{z}} \mathbf{A}_{\mathbf{z}}=-2 \mathbf{Q}_{\mathbf{z}}
$$

with $\mathbf{Q}_{\mathbf{z}}$ a positive and symmetric matrix of $\mathbb{R}^{2 \times 2}$. Choosing control law (20), the Lyapunov function time derivative is given by

$$
\dot{V}=-\lambda \quad{ }^{2}-c_{2}^{\mathrm{f}} \mathbf{Z}^{\mathrm{T}} \mathbf{Q}_{\mathrm{z}} \mathbf{Z}-\lambda_{q} \delta_{q}^{2} \leq 0
$$

that ensures GAS of the origin of the closed-loop system. At that point the convergence to zero of $\psi, \mathbf{Z}$ and $\delta_{q}$ is ensured. Thanks to the changes of coordinates (16),(18) and (21), the flexible states $N$ and the pitch rate $q$ also converge to zero.

Remark 3. When $c_{2}^{\mathrm{f}}=0$, one gets the classical backstepping controller. (In this case, the term $\mathbf{C}_{\mathbf{N}} N$ represents an exogenous disturbance which must be dominated. Note that such an unmeasured disturbance may even destabilize the system). When $c_{2}^{\mathrm{f}}>0$, this controller is referred to as flexible backstepping since it clearly acts on the oscillations of the dynamics associated with the underactuated degree of freedom. Indeed, (24) yields

$$
c_{2}^{\mathrm{f}} \mathbf{Z}^{\mathrm{T}} \mathbf{Q}_{\mathbf{z}} \mathbf{Z} \leq-\dot{V}
$$

By integration one gets

$$
\int_{0}^{+\infty} \mathbf{Z}^{\mathrm{T}}(s) \mathbf{Q} \mathbf{z}(s) \mathrm{d} s \leq \frac{1}{c_{2}^{\mathrm{f}}} V(0)
$$

This means that increasing $c_{2}^{\mathrm{f}}$ enables to limit the oscillations of the dynamics of $\mathbf{Z}$. Coming back to the original system (13), one can check by numerical simulations that the control law adds damping to the dynamics of the flexible mode $\eta$.

Remark 4. This "flexible backstepping" controller $\beta_{\mathrm{f}}$ depends on the full state and requires the knowledge of the unknown parameters. To better understand this fact, we propose to note this control law as follows

$$
\beta_{f}:=\beta_{f}(\psi, q, \mathbf{N},-2 \xi \omega,-\bar{\omega})
$$

where $\bar{\omega}=\omega^{2}-h_{\mathrm{T}} r_{\mathrm{T}} T$.

Indeed, $\beta_{f}$ depends on $\xi$ and $\omega$ since it depends on $\mathbf{P}_{\mathbf{z}}$, which in turn depends on $\mathbf{A}_{\mathbf{z}}(-2 \xi \omega,-\bar{\omega})$ through the 
relation (23). To ensure all control objectives and make the use of this law possible, we propose a dedicated estimation scheme in the following section.

\section{Main result: An adaptive finite time observer design}

Since the bending mode is not measured and flexible parameters are generally distorted, we develop in this section a method to estimate flexible states and parameters and so to make the use of our nonlinear control law possible.

\section{I. State observer design with known parameters (ideal case)}

Flexible states $\eta$ and $\dot{\eta}$ must be estimated. They are described by linear differential equations which result from system (13). Since outputs (12) involve both rigid and flexible states, the idea is to consider the augmented state $\mathbf{X}=\left(\begin{array}{lll}\psi & \eta & \dot{\eta}\end{array}\right)^{\mathrm{T}}$ instead of just the required flexible states. It is important to note that this system is linear, contrary to the original nonlinear one (13).

Working with this augmented state gives the possibility to design a linear observer, by focusing on

$$
\left\{\begin{array}{l}
\dot{\mathbf{X}}=\mathbf{A X}+\mathbf{B}_{\mathbf{y}} y_{2}+\mathbf{B}_{\beta} \beta \\
\mathbf{y}_{\mathbf{1}}=\mathbf{C X}
\end{array}\right.
$$

where $y_{2}$ acts as an input and

$$
\begin{aligned}
& \mathbf{A}=\left(\begin{array}{ccc}
0 & 0 & -r_{g y} \\
0 & 0 & 1 \\
0 & -\bar{\omega}^{2} & -2 \xi \omega
\end{array}\right) \quad \mathbf{B}_{\mathbf{y}}=\left(\begin{array}{l}
1 \\
0 \\
0
\end{array}\right) \\
& \mathbf{B}_{\beta}=\left(\begin{array}{c}
0 \\
0 \\
h_{\mathrm{T}} T
\end{array}\right) \quad \mathbf{C}=\left(\begin{array}{lll}
1 & r_{\mathrm{ci}} & 0
\end{array}\right)
\end{aligned}
$$

with $\bar{\omega}=\omega^{2}-h_{\mathrm{T}} r_{\mathrm{T}} T$.

Observability conditions hold for the pair $(\mathbf{A}, \mathbf{C})$ and estimated state $\hat{\mathbf{X}}=\left(\begin{array}{lll}\hat{\psi} & \hat{\eta} & \hat{\dot{\eta}}\end{array}\right)^{\mathrm{T}}$ is given by the classical Luenberger observer

$$
\dot{\hat{\mathbf{X}}}=\mathbf{A} \hat{\mathbf{X}}+\mathbf{B}_{\mathbf{y}} y_{2}+\mathbf{B}_{\beta} \beta+\mathbf{L}_{\mathbf{0}}\left(y_{1}-\mathbf{C} \hat{\mathbf{X}}\right)
$$

where $\mathbf{L}_{\mathbf{o}} \in \mathbb{R}^{3 \times 1}$ is chosen such that $\mathbf{A}-\mathbf{L}_{\mathbf{0}} \mathbf{C}$ is Hurwitz.

\subsection{Parameters estimation}

Bending mode natural damping and pulsation are generally subject to uncertainties and variations during flight. Besides, since our flight control law intends to attenuate the oscillations of the bending mode - that is, to add damping - it is important to know these parameters accurately.

Moreover, the accuracy and convergence time of the state observer can be improved by a better knowledge of the initial state conditions.

In this way we choose to estimate the following parameters

$$
\theta=\left(\begin{array}{c}
\theta_{1} \\
\theta_{2} \\
\theta_{3} \\
\theta_{4}
\end{array}\right)=\left(\begin{array}{c}
-2 \xi \omega \\
-\bar{\omega}^{2} \\
y_{3}(0) \\
\dot{y}_{3}(0)-\dot{y}_{2}(0)+2 \xi \omega y_{3}(0)
\end{array}\right)
$$

where $y_{3}=\dot{y}_{1}=y_{2}+\left(r_{c i}-r_{g y}\right) \dot{\eta}$.

The first two parameters $\left(\theta_{1}\right.$ and $\left.\theta_{2}\right)$ give the natural damping and pulsation of the bending mode. The flexible initial state conditions depend on the four parameters $\theta$

$$
\begin{aligned}
& \eta(0)=\frac{1}{\theta_{2}}\left(\frac{\theta_{4}+\theta_{1} y_{2}(0)}{r_{\mathrm{ci}}-r_{g y}}-h_{\mathrm{T}} T \beta(0)\right) \\
& \dot{\eta}(0)=\frac{\theta_{3}-y_{2}(0)}{r_{c i}-r_{g y}}
\end{aligned}
$$

$r_{\mathrm{ci}}$ being small, we use the first initial output to approximate the state initial condition (IC) $\psi(0)$

$$
\psi(0)=y_{1}(0)-r_{\mathrm{ci}} \eta(0) \simeq y_{1}(0)
$$

The flexible initial state conditions must be estimated through the outputs and their time derivatives. Noting that $\dot{\eta}=\frac{\dot{y}_{1}-y_{2}}{r_{\mathrm{ci}}-r_{g v}}$, it is the following equation that links the outputs and their time derivatives to the input derivative, and consists in the basic equation of the parameter estimation

$$
y_{1}^{(3)}=\theta_{2}\left(\dot{y}_{1}-y_{2}\right)+\theta_{1}\left(\ddot{y}_{1}-\dot{y}_{2}\right)+h_{\mathrm{T}} T\left(r_{\mathrm{ci}}-r_{g y}\right) \dot{\beta}+\ddot{y}_{2}
$$

An algebraic methodology for parameter identification is described in Fliess and Sira-Ramirez (2003). This approach was used here to estimate $\theta$. The main steps are presented here.

1. Take the Laplace transformation of (32) to reveal the four parameters to be estimated. So

$$
\begin{aligned}
& \theta_{1}\left[-s^{2} Y_{1}+s\left(Y_{2}+y_{1}(0) s\right)-y_{2}(0)\right] \\
& \quad+\theta_{2}\left[-s Y_{1}+Y_{2}+y_{1}(0)\right]-s \theta_{3}-\theta_{4}
\end{aligned}
$$




$$
\begin{aligned}
= & h_{\mathrm{T}} T\left(r_{\mathrm{ci}}-r_{g y}\right)[s B-\beta(0)]-s^{3} Y_{1} \\
& +s^{2}\left[Y_{2}+y_{1}(0)\right]-s y_{2}(0)
\end{aligned}
$$

where $s$ represents the Laplace variable and $Y_{i}$ (resp. B) is the Laplace transformation of signal $y_{i}(i \in\{1,2\})$ (resp. $\beta$ ).

2. Take derivatives with respect to $s$, (three times) to get unknown parameters as equations.

3. Multiply by $s^{-3}$ both sides to avoid time derivations.

4. Come back to the time domain using inverse transformations.

5. Note that the system of time-varying linear equations can be expressed in matrix form as

$$
\mathbf{P}(t) \theta=\mathbf{Q}(t)
$$

where $\mathbf{P} \in \mathbb{R}^{4 \times 4}$ and $\mathbf{Q} \in \mathbb{R}^{4 \times 1}$ (details of the matrices in Appendix 1).

The usual way to conclude the algebraic estimation technique is to use the inverse of matrix $P$ to define $\theta$. But, as $P$ is time varying, it is likely to vanish at some instants. To avoid this problem we make the following assumption

Assumption 2. PE condition: $\exists \tau>0$ such that

$$
\forall t \geq 0, \quad \int_{t}^{t+\tau} \mathbf{P}(s)^{\mathrm{T}} \mathbf{P}(s) \mathrm{d} s>0
$$

Remark 5. In view of matrix $P$ coefficients detailed in the Appendix 1 , it is clear that the PE condition depends on outputs $y_{1}, y_{2}$ and on the input $\beta$ of the system.

Under Assumption 2, $\theta$ is finally given by

$$
\theta=\left(\int_{t}^{t+\tau} \mathbf{P}(s)^{\mathrm{T}} \mathbf{P}(s) \mathrm{d} s\right)^{-1}\left(\int_{t}^{t+\tau} \mathbf{P}(s)^{\mathrm{T}} \mathbf{Q}(s) \mathrm{d} s\right)
$$

Since $\tau$ is unknown, we compute estimated parameters $\hat{\theta}$ and then $\hat{\mathbf{A}}$ and $\hat{\mathbf{X}}_{\mathbf{0}}$ at discrete time instants $\tau_{k}$ where $\left\{\tau_{k}\right\}_{k=0}^{+\infty}$ is a partition of $\mathbb{R}^{+}$, using

$$
\begin{gathered}
\hat{\theta}\left(\tau_{k}\right)=\left(\int_{0}^{\tau_{k}} \mathbf{P}(s)^{\mathrm{T}} \mathbf{P}(s) \mathrm{d} s\right)^{-1}\left(\int_{0}^{\tau_{k}} \mathbf{P}(s)^{\mathrm{T}} \mathbf{Q}(s) \mathrm{d} s\right) \\
\hat{\mathbf{A}}\left(\tau_{k}\right)=\hat{\mathbf{A}}_{\mathbf{k}}=\left(\begin{array}{ccc}
0 & 0 & -r_{g y} \\
0 & 0 & 1 \\
0 & \hat{\theta}_{2}\left(\tau_{k}\right) & \hat{\theta}_{1}\left(\tau_{k}\right)
\end{array}\right) \\
\hat{\mathbf{X}}_{\mathbf{0}}\left(\tau_{k}\right)=\left(\begin{array}{c}
y_{1}(0) \\
\frac{1}{\hat{\theta}_{2}\left(\tau_{k}\right)}\left(\frac{\hat{\theta}_{4}\left(\tau_{k}\right)+\hat{\theta}_{1}\left(\tau_{k}\right) y_{2}(0)}{r_{\mathrm{ci}}-r_{\mathrm{gy}}}-h_{\mathrm{T}} T \beta(0)\right) \\
\frac{\hat{\theta}_{3}\left(\tau_{k}\right)-y_{2}(0)}{r_{\mathrm{ci}}-r_{\mathrm{gy}}}
\end{array}\right)
\end{gathered}
$$

Remark 6. It is important to note that $\hat{\mathbf{A}}$ and $\hat{\mathbf{X}}_{\mathbf{0}}$ are not completely described by estimated parameters. Parameters $r_{\mathrm{gy}}, r_{\mathrm{ci}}, h_{T}$ and $T$ are supposed to be known, as stated in Assumption 1.

\subsection{Adaptive finite-time observer}

Mixing the results of the last subsections, it is now possible to design an observer where the parameters are unknown.

Estimated parameters and IC are used to improve the accuracy of the observer. In particular, $\hat{\theta}_{1}$ and $\hat{\theta}_{2}$ are used in the design such that the observer no longer depends on the natural damping and pulsation of the bending mode, that are subject to variations.

Proposition 2. Under Assumption 2, the following hybrid observer converges in finite-time

$$
\left\{\begin{array}{l}
\forall t \in\left[\tau_{k}, \tau_{k+1}[\right. \\
\dot{\hat{\mathbf{X}}}=\hat{\mathbf{A}}_{\mathbf{k}} \hat{\mathbf{X}}+\mathbf{B}_{\mathbf{y}} y_{2}+\mathbf{B}_{\beta} \beta+\mathbf{L}_{\mathbf{k}}\left(y_{1}-\mathbf{C} \hat{\mathbf{X}}\right) \\
\text { when } t=\tau_{k} \\
\hat{\mathbf{X}}\left(\tau_{k}\right)=\mathbf{e}^{\hat{\mathbf{A}}_{k} \tau_{\mathbf{k}}} \hat{\mathbf{X}}_{\mathbf{0}}\left(\tau_{k}\right)+\int_{0}^{\tau_{k}} \mathbf{e}^{\hat{\mathbf{A}}_{\mathbf{k}}\left(\tau_{k}-\mathbf{s}\right)}\left[\mathbf{B}_{\mathbf{y}} y_{2}(s)+\mathbf{B}_{\beta} \beta(s)\right] \mathrm{d} s
\end{array}\right.
$$

At each $\tau_{k}$, the state is updated and the dynamics $\left(\hat{A}_{k}\right)$ as well.

$\mathbf{L}_{\mathbf{k}}$ is chosen such that $\hat{\mathbf{A}}_{\mathbf{k}}-\mathbf{L}_{\mathbf{k}} \mathbf{C}$ is Hurwitz.

Proof. Using the notations $\tilde{\mathbf{X}}=\mathbf{X}-\hat{\mathbf{X}}$ and $\tilde{\mathbf{A}}=\mathbf{A}-\hat{\mathbf{A}}_{k}$, it comes

$$
\dot{\tilde{\mathbf{X}}}=\mathbf{A} \mathbf{X}-\hat{\mathbf{A}}_{\mathbf{k}} \hat{\mathbf{X}}-\mathbf{L}_{\mathbf{k}} \mathbf{C} \tilde{\mathbf{X}}=\left(\hat{\mathbf{A}}_{\mathbf{k}}-\mathbf{L}_{\mathbf{k}} \mathbf{C}\right) \tilde{\mathbf{X}}+\tilde{\mathbf{A}} \mathbf{X}
$$

Because of the PE condition, algebraic parameter estimation converges in finite-time. Thus, there exists $k^{*}$ such that $\tau_{k^{*}}>\tau>0$ and

$$
\forall k>k^{*}, \quad \hat{\theta}\left(\tau_{k}\right)=\theta
$$

Consequently $\forall k>k^{*}, \hat{\mathbf{A}}_{\mathbf{k}}=\mathbf{A}$ and $\mathbf{L}_{\mathbf{k}}=\mathbf{L}_{\mathbf{o}}$. The estimation error satisfies

$$
\forall t \geq \tau_{k}^{*}, \quad \dot{\tilde{\mathbf{X}}}=\left(\mathbf{A}-\mathbf{L}_{\mathbf{0}} \mathbf{C}\right) \tilde{\mathbf{X}}
$$

$\mathbf{A}-\mathbf{L}_{\mathbf{0}} \mathbf{C}$ being Hurwitz, the estimation error $\tilde{\mathbf{X}}$ converges asymptotically to zero.

Besides, the estimated state $\hat{X}(t)$ is updated on $\hat{\mathbf{X}}\left(\tau_{k}\right)$ at each $\tau_{k}$ verifying $\tau_{k}>\tau_{k^{*}}$

$$
\forall t \geq \tau_{k}^{*}, \quad \hat{\mathbf{X}}_{\mathbf{0}}(\mathbf{t})=\mathbf{X}_{\mathbf{0}}(\mathbf{t}) \quad \text { ie } \quad \hat{\mathbf{X}}\left(\tau_{k}\right)=\mathbf{X}\left(\tau_{k}\right)
$$


Estimation error is then given by

$$
\forall t \geq \tau_{k}^{*}, \quad \tilde{\mathbf{X}}(t)=\tilde{\mathbf{X}}\left(\tau_{k}\right) \mathbf{e}^{\left(\mathbf{A}-\mathbf{L}_{\mathbf{0}} \mathbf{C}\right)\left(\mathbf{t}-\tau_{\mathbf{k}}\right)}=0
$$

Finally the estimation error vanishes in finite-time.

Remark 7. The proposed observer (40) was inspired by the work of Karafyllis and Jiang (2011). We've added the gain $\boldsymbol{L}_{k}$ to account for possible measurement noise. Consider that we measure $y_{1}+d_{1}$ and $y_{2}+d_{2}$ where $d_{1}$ and $d_{2}$ are measurement noise. This gives: $\forall t \in\left[\tau_{k}, \tau_{k+1}[\right.$

$$
\dot{\tilde{\mathbf{X}}}=\left(\hat{\mathbf{A}}_{\mathbf{k}}-\mathbf{L}_{\mathbf{k}} \mathbf{C}\right) \tilde{\mathbf{X}}+\tilde{\mathbf{A}} \mathbf{X}-B_{y} d_{2}-L_{k} d_{1}
$$

This is an input to state stable (ISS) system, since it can be seen as an asymptotically stable linear system driven by the input $\tilde{\mathbf{A}} \mathbf{X}-B_{y} d_{2}-L_{k} d_{1}$. Note that the term $\tilde{\mathbf{A}} \mathbf{X}$ disappears once matrix $\tilde{A}$ is correctly estimated (however, contrary to the above discussion, it might not disappear in case of noisy measurements). Note that when $L_{k}=0$, the observation error dynamics is not an ISS system since $\hat{\mathbf{A}}_{\mathbf{k}}$ is marginally stable according to equation (38).

\section{Simulation results}

\section{I. Parameters estimator}

Figure 3 shows in blue (resp. in red) the evolution of flexible estimated parameters $\left(\hat{\theta}_{1}, \hat{\theta}_{2}\right)$ and initial state conditions $\hat{\mathbf{X}}_{\mathbf{0}}=\left(\begin{array}{lll}\hat{\psi}(0) & \hat{\eta}(0) & \hat{\dot{\eta}}(0)\end{array}\right)$ when the sensors give ideal (resp. noisy) measurements. The red curve was obtained applying a zero-mean periodic noise on signals $y_{1}$ and $y_{2}$. The simulation has been performed with the following parameters

$$
\begin{aligned}
\theta_{1} & =-1.2 \quad \theta_{2}=-35 \quad \psi(0)=20^{\circ} \\
\eta(0) & =10 \quad \dot{\eta}(0)=5
\end{aligned}
$$

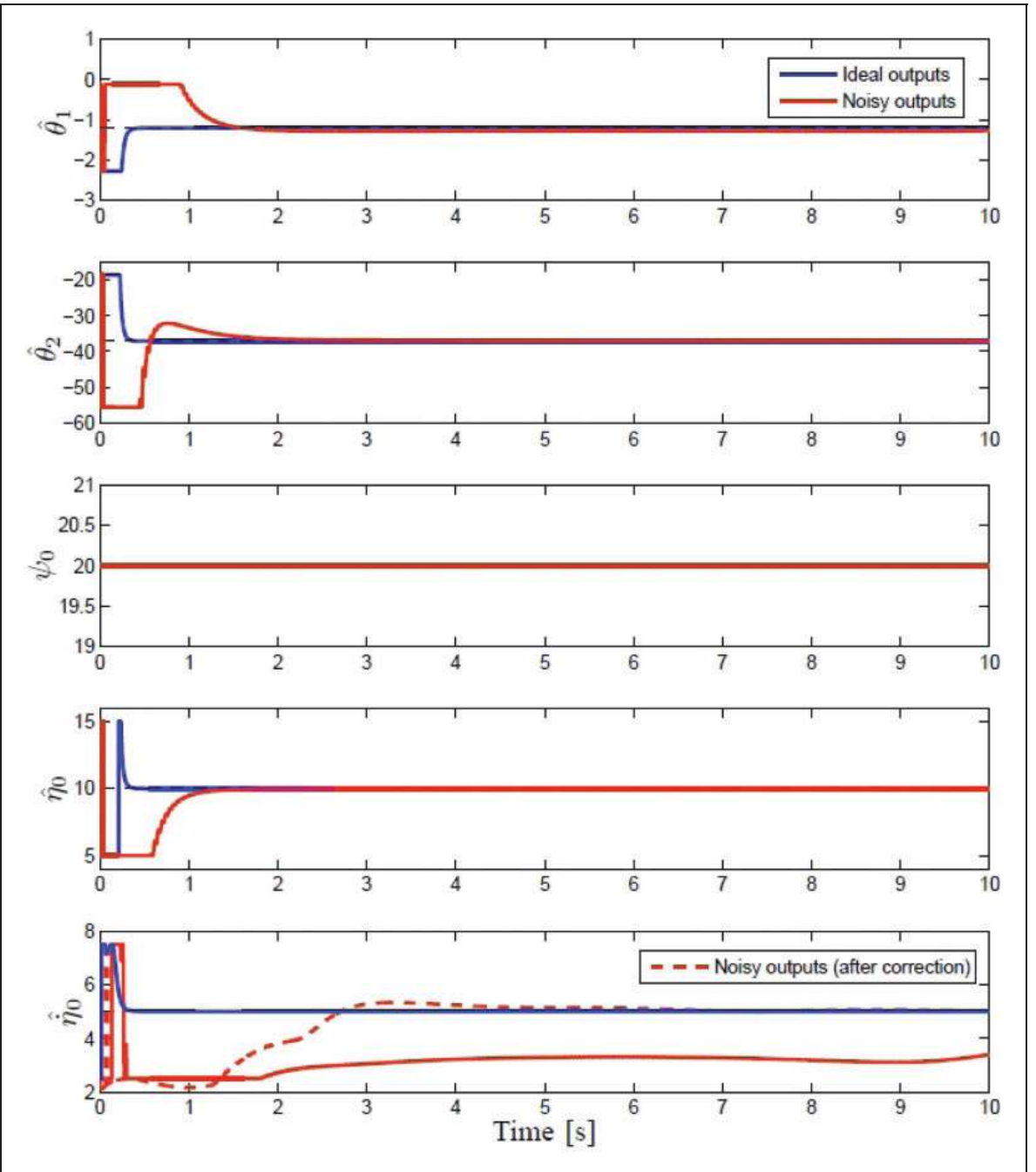

Figure 3. Estimated parameters. 
Remark 8. With noisy measurements, estimated parameter $\hat{\dot{\eta}}(0)$ is biased and the estimation error $\tilde{\dot{\eta}}=$ $\dot{\eta}(0)-\dot{\eta}(0)$ is quite important (about 1.5). This problem is detailed in the following subsection.

\subsection{Robustness improvement of the observer with respect to noisy measurements}

An estimation bias appears in simulation on estimated parameter $\hat{\dot{\eta}}(0)$ when measurements are noisy (solid red curve on the last plot of Figure 3). It is given by

$$
\tilde{\dot{\eta}}(0)=\dot{\eta}(0)-\hat{\dot{\eta}}(0)=\frac{\theta_{3}-\hat{\theta}_{3}}{r_{c i}-r_{g y}}
$$

This bias term is due to the low values of $r_{c i}-r_{g y}$ which accentuates the (low) difference between $\theta_{3}$ and $\hat{\theta}_{3}$. This section proposes a way to identify this estimation bias $\tilde{\dot{\eta}}(0)$ and thus to correct $\hat{\mathbf{X}}_{\mathbf{0}}\left(\tau_{k}\right)$.

From system (28), we have

$$
y_{1}(t)=\mathbf{C e}^{\mathbf{A t}} \mathbf{X}_{\mathbf{0}}(t)+\mathbf{C} \int_{0}^{t} \mathbf{e}^{\mathbf{A}(\mathbf{t}-\mathbf{s})}\left[\mathbf{B}_{\mathbf{y}} y_{2}(s)+\mathbf{B}_{\beta} \beta(s)\right] \mathrm{d} s
$$

Similarly, with the estimated state

$$
\hat{\mathbf{X}}(t)=\mathbf{e}^{\hat{\mathbf{A}}_{\mathbf{k}}} \mathbf{\mathbf { X } _ { \mathbf { 0 } }}(t)+\int_{0}^{t} \mathbf{e}^{\hat{\mathbf{A}}_{\mathbf{k}}(\mathbf{t}-\mathbf{s})}\left[\mathbf{B}_{\mathbf{y}} y_{2}(s)+\mathbf{B}_{\beta} \beta(s)\right] \mathrm{d} s
$$

Under the PE condition, algebraic parameter estimation still converges in finite-time. Thus, there exists $k^{*}>0$ such that for all $k \geq k^{*}$

$$
\hat{\mathbf{A}}_{\mathbf{k}}=\mathbf{A} \quad \text { and } \quad \hat{\mathbf{X}}_{\mathbf{0}}=\left(\begin{array}{lll}
\psi(0) & \eta(0) \quad \dot{\eta}(0)-\tilde{\dot{\eta}}(0)
\end{array}\right)^{\mathrm{T}}
$$

This last equation underlines the fact that only the estimated IC $\dot{\eta}$ is biased. Then

$$
\forall t>t_{k^{*}} \quad y_{1}(t)-\mathbf{C} \hat{\mathbf{X}}(t)=\mathbf{C e}^{\mathbf{A t}} \mathbf{e}_{\mathbf{3}} \tilde{\dot{\eta}}(0)
$$

with $\mathbf{e}_{3}=\left(\begin{array}{lll}0 & 0 & 1\end{array}\right)^{\mathrm{T}}$. That can be written as

$$
\int_{0}^{t}\left(\mathbf{C e}^{\mathbf{A s}} \mathbf{e}_{3}\right)^{2} \mathrm{~d} s \tilde{\dot{\eta}}(0)=\int_{0}^{t} \mathbf{C e}^{\mathbf{A s}} \mathbf{e}_{3}\left[y_{1}(s)-\mathbf{C} \hat{\mathbf{X}}(s)\right] \mathrm{d} s
$$

Supposing that $\int_{0}^{t}\left(\mathbf{C e}^{\mathbf{A s}} \mathbf{e}_{3}\right)^{2} \mathrm{~d} s \neq 0$, estimation error is given by

$$
\tilde{\eta}(0)=\frac{\int_{0}^{t} \mathbf{C e}^{\mathbf{A s}} \mathbf{e}_{3}\left[y_{1}(s)-\mathbf{C} \hat{\mathbf{X}}(s)\right] \mathrm{d} s}{\int_{0}^{t}\left(\mathbf{C e}^{\mathbf{A s}} \mathbf{e}_{3}\right)^{2} \mathrm{~d} s}
$$

This term is added on the last simulation. The result is represented by the dashed red line on Figure 3.

\subsection{Finite time observer}

Figure 4 compares this finite-time observer that updates the state from the knowledge of the IC (in blue) with the same Luenberger observer without any update of the estimated state (in green). In the two cases, the observer parameter $\mathbf{L}_{\mathbf{o}}$ is the same. Moreover, the ICs of the classical Luenberger observer have been chosen very close to the estimated parameters, so that the comparison is fair. This figure shows how the observer convergence time is improved. The dramatic slope on the blue curve corresponds to the moment where the estimated state is updated.

\section{Closed-loop simulations}

In this section, the adaptive finite-time observer is blended with the flexible backstepping law and closedloop simulations are realized for separate locations of sensors.

\section{I. Closed-loop system}

Before reaching the convergence time $\tau_{k}^{*}$, asymptotic stability of the closed-loop system, composed of system (17) and the flexible backstepping law, is not guaranteed. For that reason, the final control law is designed as a switch between:

- classical backstepping law applied to the sole rigid dynamics and consisting of an output-feedback controller, assuming that $\psi$ (resp. $q$ ) approximately corresponds to output $y_{1}$ (resp. $y_{2}$ ), before reaching convergence time;

- flexible backstepping controller using estimated flexible states and parameters as soon as convergence time is reached.

Using the notation (27), this switching controller is summarized as follows

$$
\beta=\left\{\begin{array}{l}
\beta_{c}\left(y_{1}, y_{2}\right) \quad \text { when } t<\tau_{k}^{*} \\
\hat{\beta}_{\mathrm{f}}:=\beta_{\mathrm{f}}\left(y_{1}-r_{c i} \hat{\eta}, y_{2}-r_{g y} \hat{\dot{\eta}}, \hat{\mathbf{N}}, \hat{\theta}_{1}, \hat{\theta}_{2}\right) \text { else }
\end{array}\right.
$$




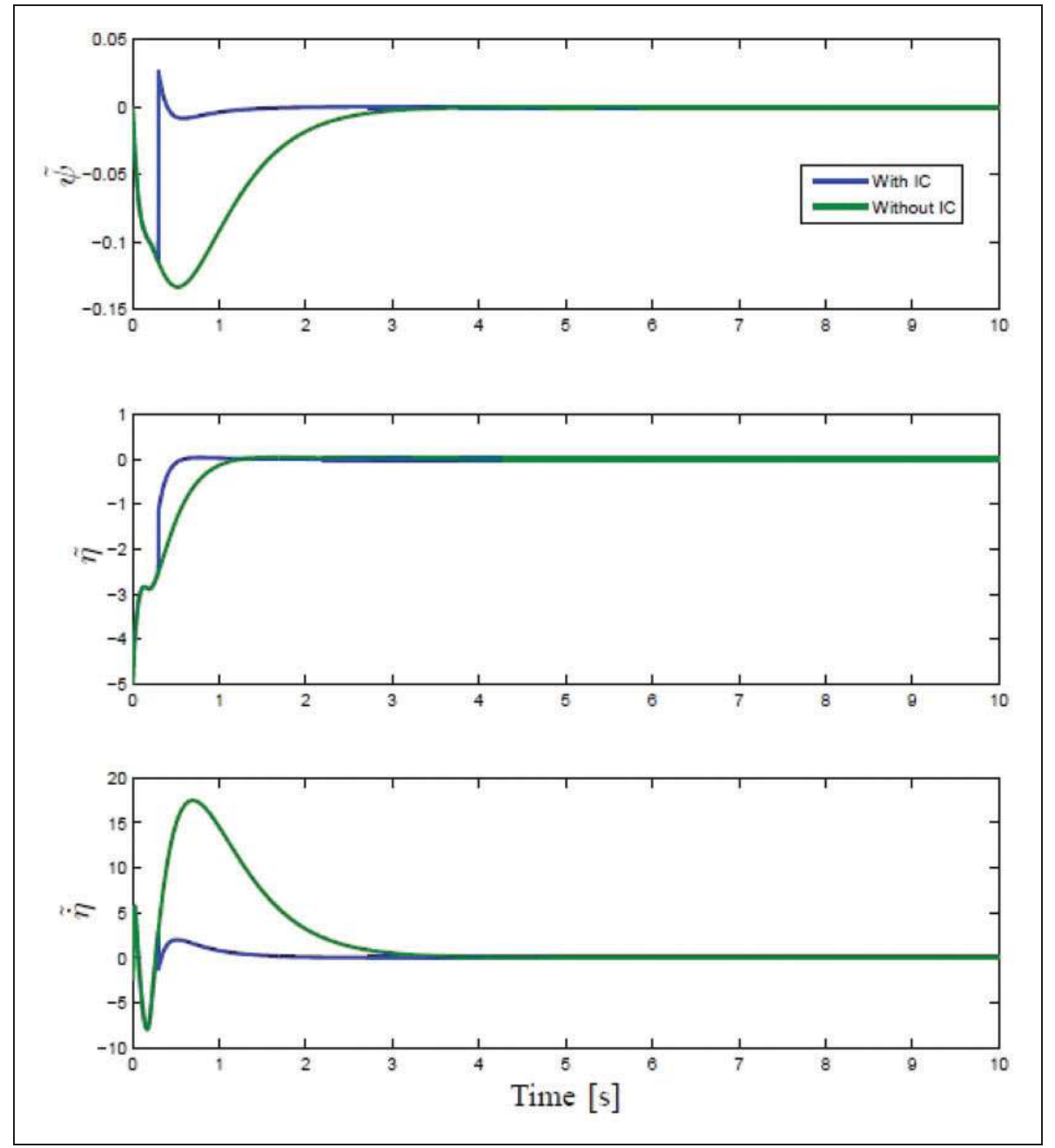

Figure 4. Observation errors.

$\beta^{c}$ is the backstepping controller designed on the sole rigid-dynamics, which is in strict-feedback form (Duraffourg et al., 2013c). This control law corresponds to the flexible backstepping (20) without flexible states (the expression of $\beta_{c}$ is detailed hereafter (55)).

The closed-loop system is illustrated in Figure 5.

\subsection{Closed-loop simulation for a collocated rate-gyro sensor}

Collocation occurs if the dynamics between the actuator and sensor show a repeating sequence of antiresonances and resonances, with the consequence that the phase never drops below $-180^{\circ}$, which is beneficial for stability. For noncollocated actuators and sensors this repeating sequence is not guaranteed (for more details see, for instance, Frosch and Vallely, 1967; Spector and Flashner, 1990; Spong, 1996; OlfatiSaber, 2000; Preumont, 2011).
Figure 2 illustrates the location of

1. a collocated rate-gyro (gyro "a"): $y_{2}=q+r_{g y}^{a} \dot{\eta}$

2. a noncollocated rate-gyro (gyro "b"): $y_{2}=$ $q+r_{g y}^{b} \dot{\eta}$

This study only considered the collocated case for two reasons:

1. The following assumption was numerically validated:

Assumption 3. the linearized closed-loop system has all its eigenvalues with strictly negative real parts.

2. Using assumptions 1 and 3 , it is possible to prove that the closed-loop system (13)-(54) possesses (at least) a region of attraction around zero. (This is discussed in the following section.) 


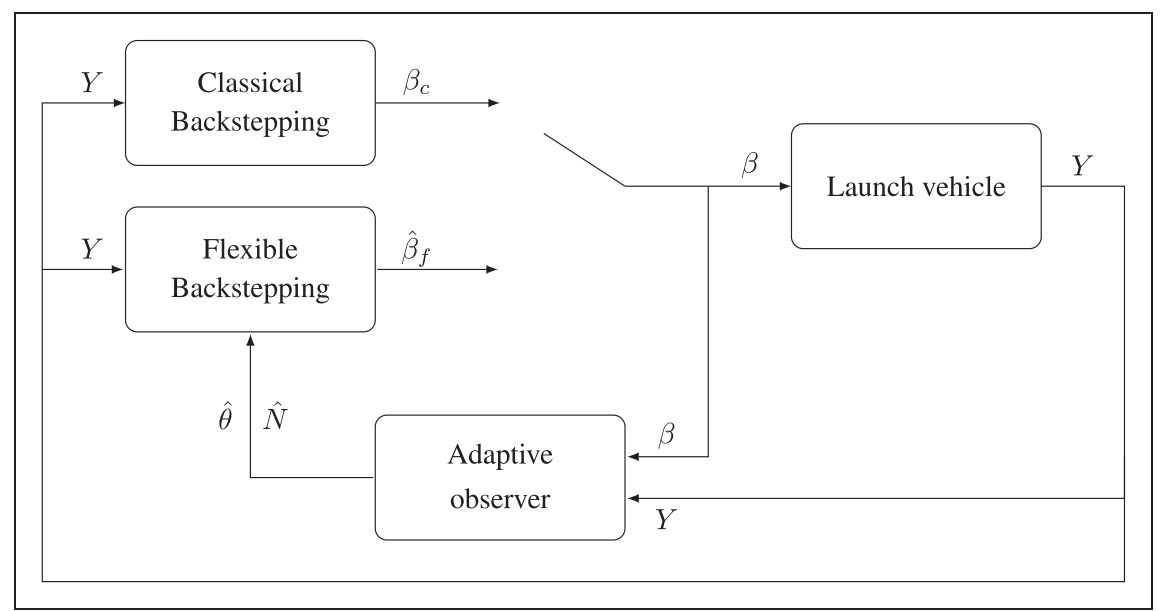

Figure 5. Block diagram of the closed-loop system.

The switching controller (54) is now denoted by Observer Flexible Backstepping. It was compared with:

- the "Classical backstepping control" law $\beta^{c}$ (54) which use the ouputs $y_{1}$ and $y_{2}$;

- an ideal full-state feedback flexible backstepping law given by (20), assuming the flexible states $\mathbf{N}$ and the parameters $\xi$ and $\omega$ are known.

This last controller has demonstrated good performance in terms of stability and bending mode damping. Consequently, it corresponds to a reference law and enables us to judge the efficiency of the designed switching output-feedback controller.

Figure 6 shows the evolution of attitude, pitch rate and flexible states with time. The simulation was performed with natural damping equal to 0.005 and a natural frequency equal to $6 \mathrm{rad} \cdot \mathrm{s}^{-1}$. Control parameters have been tuned so that the time responses are the same.

The three controllers converge asymptotically to the origin.

As expected (see Remark 3), full state-feedback flexible backstepping improves the damping of the bending mode compared to classical backstepping. Moreover, it is remarkable that observer flexible backstepping is able to damp out the bending mode when the flexible states are unmeasured and flexible parameters $\xi$ and $\omega$ are uncertain.

\subsection{Further discussions on the estimation phase}

6.3.I. Closed-loop stability. Thanks to the finite time convergence property of the observer (40), the controller defined by (54) switches once at $t=\tau_{k}^{*}$. Such a switch occurs when the parameters $\theta$ (30) have converged.

The closed-loop system defined by (17), (40) and (54) inherits the GAS property proved in Proposition 1 when $t \geq \tau_{k}^{*}$. Meanwhile, it is necessary to prove that this closed-loop system remains stable for $t \in\left[0, \tau_{k}^{*}[\right.$.

Let us consider system (13).

For $t \in\left[0, \tau_{k}^{*}[\right.$, the following feedback control law is applied

$\beta_{c}\left(y_{1}, y_{2}\right)=\frac{c_{\beta}}{h_{\mathrm{T}} T}\left[-\frac{1}{c_{1}^{\mathrm{f}}} \lambda_{\psi} y_{2}+\frac{l_{\text {aero }}}{I_{L}} L\left(y_{1}\right)-\frac{1}{c_{3}^{\mathrm{f}}}\left(c_{1}^{f} y_{1}+\lambda_{q} \delta_{y_{2}}\right)\right]$

with tuning gains $\lambda_{q}>0, \lambda_{\psi}>0, c_{i}^{f} \quad i \in\{1,2\}$ and where

$$
y_{2, c m d}=-\frac{1}{c_{1}^{\mathrm{f}}} \lambda_{\psi} y_{1} ; \quad \delta_{y_{2}}=y_{2}-y_{2, c m d}
$$

Considering equations (12), (14) and (55), it is clear that

$$
\beta_{c}\left(y_{1}, y_{2}\right)=\beta_{c}(\psi, q)+\mathbf{C}_{\beta} \mathbf{N}+g\left(\psi \eta, \eta^{2}\right)
$$

where

$$
\begin{gathered}
\mathbf{C}_{\beta}=-\frac{c_{\beta}}{h_{\mathrm{T}} T}\left(\frac{1}{c_{3}^{\mathrm{f}}}\left(c_{1}^{\mathrm{f}}+\frac{\lambda_{\psi} \lambda_{q}}{c_{3}^{\mathrm{f}}}\right) r_{c i}\left(\frac{\lambda}{c_{1}^{\mathrm{f}}}+\frac{\lambda_{q}}{c_{3}^{\mathrm{f}}}\right) r_{g y}\right) \\
g\left(\psi \eta, \eta^{2}\right)=-\frac{c_{\beta} l_{a e r o}}{h_{\mathrm{T}} T I_{L}} \bar{q} S C_{L}^{2} r_{c i}\left(2 \psi \eta+r_{c i} \eta^{2}\right)
\end{gathered}
$$

Therefore, the closed-loop system (13)-(20) is rewritten as follows

$$
\left\{\begin{array}{l}
\dot{\psi}=q \\
\dot{q}=-\frac{l_{\text {aero }}}{I_{L}} L(\psi)+\mathbf{C}_{\mathbf{N y}} \mathbf{N}+\mathbf{d}_{\mathbf{N}}\left(\beta_{c}(\psi, q)+g\left(\psi \eta, \eta^{2}\right)\right) \\
\dot{\mathbf{N}}=\mathbf{A}_{N y} \mathbf{N}+\mathbf{B}_{N}\left(\beta_{c}(\psi, q)+g\left(\psi \eta, \eta^{2}\right)\right)
\end{array}\right.
$$




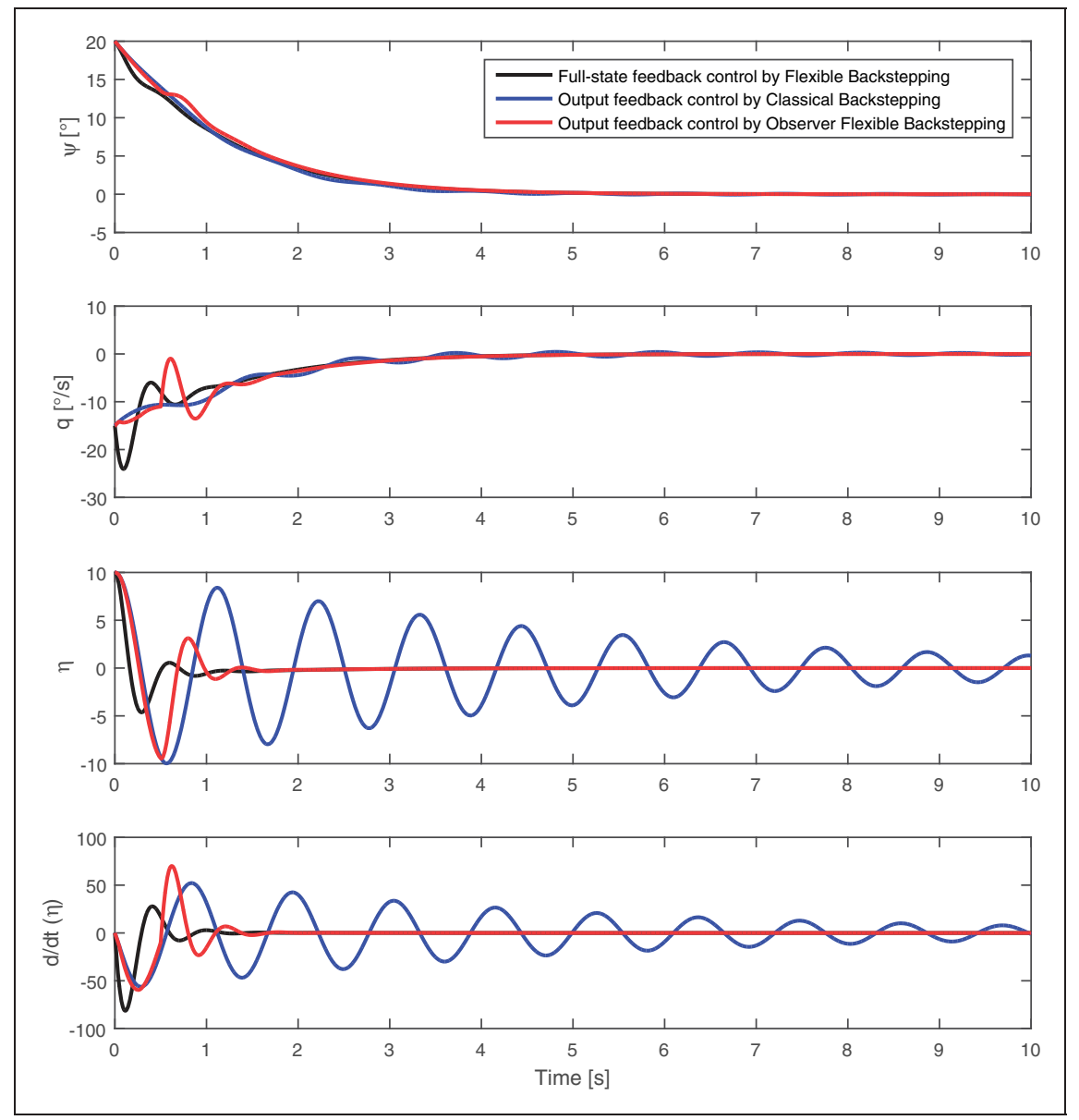

Figure 6. Closed-loop comparison.

where

$$
\mathbf{A}_{\mathrm{Ny}}=\mathbf{A}_{N}+\mathbf{B}_{N} \mathbf{C}_{\beta} ; \quad \mathbf{C}_{\mathbf{N y}}=\mathbf{C}_{\mathbf{N}}+\mathbf{d}_{\mathbf{N}} \mathbf{C}_{\beta}
$$

\section{Discussion:}

1. Let us suppose assumptions 1 and 3 hold. The closed-loop system (60) is Locally Asymptotically Stable by virtue of Lyapunov's indirect method (for more details, see Theorem 3.7 in Khalil (1996). In other words, this means a region of attraction exists around zero.

2. Consider:

\section{Assumption 4.}

$$
r_{c i}=0
$$

In this case the nonlinear term $\left.g\left(\psi \eta, \eta^{2}\right)\right)$ disappears! Under Assumptions 1, 3 and 4 (plus some mild assumptions) one can apply the results of Arcak and Kokotovic (2000) to redesign the backstepping controller $\beta_{c}$ and prove GAS of the closed-loop system (60)
(For more details, see Theorem 1 in Arcak and Kokotovic, 2000.)

3. Finally, when $r_{c i} \neq 0$ and when Assumptions 1 and 3 hold, the aforementioned redesigned controller might not be able to globally stabilize system (60) because of the nonlinear term $g\left(\psi \eta, \eta^{2}\right)$ ) (which is also changed by the redesign technique). In this case, a region of attraction might be computed. Future research may be devoted to show that the estimation of the region of attraction is enlarged once the backstepping controller $\beta_{c}$ is redesigned. This point will be addressed in the future.

6.3.2. Closed-loop observability. As remarked before (see Remark 5), Assumption 2 depends on the trajectories followed by $y_{1}(t)$ and $y_{2}(t)$. (It also depends on $\beta_{c}$, which in turn depends on $y_{1}(t)$ and $y_{2}(t)$ in a closed loop)

Thus, another problem consists of using the observer (40) within the transition phase of the closed-loop system (17), (54). Indeed, one must guarantee that the 
excitation condition (35) is satisfied. Note that this PE condition is our Assumption 2, which can reasonably be made in open loop. In our closed-loop simulations, this condition was respected.

However, when this assumption is not satisfied, it is necessary to make the system outputs $y_{1}(t), y_{2}(t)$ track sufficiently exciting reference signals $y_{1}^{r}(t), y_{2}^{r}(t)$ (which are set to 0 when $t \geq \tau_{k}^{*}$ ).

Few results deal with the link between the design of such exciting reference signals and the closed-loop PE condition. For a further discussion on this topic, the interested reader is referred to Adetola and Guay (2006), Adetola and Guay (2008) and the references therein.

This is another possible future research direction.

\section{Conclusion}

A mathematical model for a flexible launch vehicle has been formulated, using the Lagrange mechanism and a free-free Euler-Bernoulli beam model. Next, a full state flexible backstepping controller, which both guarantees GAS of the closed-loop nonlinear system and improves the bending mode damping, was proposed. Then, an adaptive finite-time observer that enables estimation of flexible states with unknown flexible parameters has been specifically designed in order to blend with this control design method. Numerical simulations have been performed in the case of collocation between sensors and actuator: They have clearly shown the efficiency of the designed controller in conjunction with the proposed observer.

For our future work, uncertainties on mode shapes and aerodynamics coefficients will be considered. Moreover, this novel work on using a model of the flexible launch vehicle possessing a nonlinear aerodynamic coefficient in the control design has raised several issues which need further investigation: For example, the estimation of the closed-loop system's region of attraction, the use of noncollocated outputs, and the consideration of several flexible modes. Finally, we plan to apply the flexible backstepping design method to a highly representative flexible satellite simulator. We would also like to experimentally test this method on a flexible robotic arm.

\section{Declaration of Conflicting Interests}

The author(s) declared no potential conflicts of interest with respect to the research, authorship, and/or publication of this article.

\section{Funding}

The author(s) received no financial support for the research, authorship, and/or publication of this article.

\section{References}

Adetola V and Guay M (2006) Excitation signal design for parameter convergence in adaptive control of linearizable systems. In: Proceedings of the 45th IEEE conference on decision and control, San Diego, CA, pp.447-452. .

Adetola V and Guay M (2008) Finite-time parameter estimation in adaptive control of nonlinear systems. IEEE Transactions on Automatic Control 53(3): 807-811.

Arcak M and Kokotovic PV (2000) Robust nonlinear control of systems with input unmodeled dynamics. Systems \& Control Letters 41(2): 115-122.

Ahmed-Ali T and Lamnabhi Lagarrigue F (1999) Sliding observer-controller design for uncertain triangular nonlinear systems. IEEE Transactions on Automatic Control 4(6): 1244-1249.

Boiffier JL (1998) The Dynamics of Flight: The Equations. Hoboken, NJ: Wiley.

Burlion L, Duraffourg E, Ahmed-Ali T, et al. (2013) Global asymptotic stabilization for some nonlinear models of flexible aerospace vehicles. In: Proceedings of the 52nd IEEE conference on decision and control, Florence, Italy, pp.4230-4235.

Clement B (2001) Multiobjective synthesis and gain scheduling: Application to an aerospace launcher guidance. $\mathrm{PhD}$ Dissertation, University of Paris XI, Orsay, France.

Clement B, Duc G and Mauffrey S, et al. (2001) Gain scheduling for an aerospace launcher with bending modes. In: Proceedings of the 15th IFAC symposium on automatic control in aerospace, Bologna, Italy, pp.475-480.

De Luca A (2015) Flexible robots. In: Baillieul J and Samad T (eds) Encyclopedia of Systems and Control. London: Springer, pp. 451-458.

Duraffourg E, Burlion L, Ahmed-Ali T, et al. (2013) Non-linear control of the longitudinal rotational dynamics of a flexible aircraft. In: Proceedings of the 12th European control conference, Zürich, Switzerland, pp.335-340.

Duraffourg E, Burlion L, Ahmed-Ali T, et al. (2013) Non-linear full-state control of a flexible hypersonic vehicle. In: Proceedings of the 11th IEEE international workshop on electronics control measurement signals and their application to mechatronics, Toulouse, France.

Duraffourg E, Burlion L and Ahmed-Ali T (2013) Longitudinal modeling and preliminary control of a nonlinear flexible launch vehicle. In: Proceedings of the 11th IFAC international workshop on adaptation and learning in control and signal processing, Caen, France.

Duraffourg E, Burlion L, Ahmed-Ali T, et al. (2014) Adaptive finite-time observer for a nonlinear and flexible space launch vehicle. In: Proceedings of 19th world congress of the international federation of automatic control, Cape Town, South Africa, pp.546-551.

Engel R and Kreisselmeier G (2002) A continuous-time observer which converges in finite time. IEEE Transactions on Automatic Control 47(7): 1202-1204.

Fiorentini L, Serrani A, Bolender MA, et al. (2009) Nonlinear robust adaptive control of flexible air-breathing hypersonic vehicles. Journal of Guidance, Control and Dynamics 32(2): 401-416. 
Fliess M and Sira-Ramirez H (2003) An algebraic framework for linear identification. ESAIM Control, Optimisation and Calculus of Variations 9: 151-168.

Frosch JA and Vallely DP (1967) Saturn AS-501/S-IC flight control system design. Journal of Spacecraft 4(8): 1003-1009.

Hervas JR and Reyhanoglu M (2012) Control of a spacecraft with time-varying propellant slosh parameters. In: Proceedings of the 12th international conference on control automation and systems, Jeju Island, Korea.

$\mathrm{Hu}$ Q (2009) Robust adaptive backstepping attitude and vibration control with L2-gain performance for flexible spacecraft under angular velocity constraint. Journal of Sound and Vibration 327(3-5): 285-298.

$\mathrm{Hu} \mathrm{X}, \mathrm{Wu} \mathrm{L}, \mathrm{Hu} \mathrm{C}$, et al. (2012) Adaptive sliding mode tracking control for a flexible air-breathing hypersonic vehicle. Journal of the Franklin Institute 349(2): 559-577.

Karafyllis I and Jiang ZP (2011) Hybrid dead-beat observers for a class of nonlinear systems. Systems and Control Letters 60: 608-617.

Khalil H (1996) Nonlinear Systems. Upper Saddle River, NJ: Prentice Hall.

Menold PH, Findeisen R and Allgöwer F (2003) Finite time convergent observers for nonlinear systems. In: Proceedings of the 42nd IEEE conference on decision and control, Maui, HI, pp.5673-5678.

Olfati-Saber R (2000) Trajectory tracking for a flexible onelink robot using a nonlinear noncollocated output. In: Proceedings of the 39th IEEE conference on decision and control, Sydney, Australia, pp.4024-4029.

Pan H, Sun W, Gao H, et al. (2015a) Disturbance observerbased adaptive tracking control with actuator saturation and its application. IEEE Transactions in Automation Science and Engineering 99: 1-8.

Pan H, Sun W, Gao H, et al. (2015b) Finite-time stabilization for vehicle active suspension systems with hard constraints. IEEE Transactions on Intelligent Transportation Systems 16(5): 2663-2672.

Perruquetti W, Floquet T and Moulay E (2008) Finitetime observers: Application to secure communication. IEEE Transactions on Automatic Control 53(1): 356-360.

Preumont A (2011) Vibration Control of Active Structures: An Introduction, 3rd ed. Berlin, Germany: Springer.

Spector VA and Flashner H (1990) Modelling and design implications of non-collocated control in flexible systems. ASME Journal of Dynamics Systems, Measurement and Control 11: 186-193.

Spong MW (1996) Energy based control of a class of underactuated mechanical systems. In: Proceedings of the IFAC world congress, San Francisco, CA, pp.431-436.

Shtessel YB, Baev S, Edwards C, et al. (2010) HOSM observer for a class of nonminimum phase causal nonlinear MIMO systems. IEEE Transactions on Automatic Control 55(2): 543-548.

Shtessel YB, Hall CE, Baev S, et al. (2010) Flexible modes control using sliding mode observers: Application to Ares I. In: Proceedings of the AIAA guidance, navigation and control conference, Canada, Toronto.
Sun W, Pan H and Gao H (2016) Filter-based adaptive vibration control for active vehicle suspensions with electrohydraulic actuators. IEEE Transactions in Vehicular Technology 65(6): 4619-4626.

Yu P, Shtessel Y and Edwards C (2015) Adaptive continuous higher order sliding mode control of air breathing hypersonic missile for maximum target penetration. In: $A I A A$ guidance, navigation, and control conference.

\section{Appendix I. P and $\mathbf{Q}$ matrices}

Once back in the time domain, the following relation is used to make the implementation easier (Fliess and Sira-Ramirez, 2003)

$$
\begin{gathered}
\int_{0}^{t} \int_{0}^{t_{v-1}} \cdots \int_{0}^{t_{1}} \tau^{\alpha} x(\tau) \mathrm{d} t_{v-1} \cdots \mathrm{d} t_{1} \mathrm{~d} \tau \\
=\int_{0}^{t} \frac{(t-\tau)^{v-1}}{(v-1) !} \tau^{\alpha} x(\tau) \mathrm{d} \tau
\end{gathered}
$$

Matrices $P$ and $Q$ are then expressed as

$$
\begin{gathered}
\mathbf{P}(t)=\left(\begin{array}{cccc}
P_{11}(t) & P_{12}(t) & 0 & 0 \\
P_{21}(t) & P_{22}(t) & 0 & 0 \\
P_{31}(t) & P_{32}(t) & P_{33}(t) & 0 \\
P_{41}(t) & P_{42}(t) & 0 & P_{44}(t)
\end{array}\right) \\
\mathbf{Q}(t)=\left(\begin{array}{llll}
Q_{1}(t) & Q_{2}(t) & Q_{3}(t) & \left.Q_{4}(t)\right)^{\mathrm{T}}
\end{array}\right.
\end{gathered}
$$

with

$$
\begin{aligned}
P_{11}(t)= & \int_{0}^{t}\left(-\frac{(t-s)^{3}}{3} s+\frac{(t-s)^{2}}{2} s^{2}\right) y_{2}(s) \mathrm{d} s \\
& +\int_{0}^{t}\left(\frac{(t-s)^{3}}{3}+2(t-s)^{2} s-(t-s) s^{2}\right) y_{1}(s) \mathrm{d} s \\
P_{12}(t)= & \int_{0}^{t} \frac{(t-s)^{3}}{6} s^{2} y_{2}(s) \mathrm{d} s \\
& +\int_{0}^{t}\left(\frac{(t-s)^{3}}{3} s-\frac{(t-s)^{2}}{2} s^{2}\right) y_{1}(s) \mathrm{d} s \\
P_{21}(t)= & \int_{0}^{t}\left(\frac{(t-s)^{3}}{2} s^{2}-\frac{(t-s)^{2}}{2} s^{3}\right) y_{2}(s) \mathrm{d} s \\
& +\int_{0}^{t}\left((t-s)^{3} s-3(t-s)^{2} s^{2}+(t-s) s^{3}\right) y_{1}(s) \mathrm{d} s \\
P_{22}(t)= & \int_{0}^{t}-\frac{(t-s)^{3}}{6} s^{2} y_{2}(s) \mathrm{d} s \\
& +\int_{0}^{t}\left(-\frac{(t-s)^{3}}{2} s^{2}+\frac{(t-s)^{2}}{2} s^{3}\right) y_{1}(s) \mathrm{d} s
\end{aligned}
$$




$$
\begin{aligned}
& P_{31}(t)=\int_{0}^{t}\left(\frac{(t-s)^{4}}{24}+\frac{(t-s)^{3}}{6} s-\frac{(t-s)^{2}}{2} s^{2}\right) y_{2}(s) \mathrm{d} s \\
& +\int_{0}^{t}\left(\frac{-3(t-s)^{2}}{2} s+(t-s) s^{2}\right) y_{1}(s) \mathrm{d} s+\frac{t^{4}}{24} y_{1}(0) \\
& Q_{3}(t)=-h T\left(r_{\mathrm{ci}}-r_{\mathrm{gy}}\right) \int_{0}^{t}\left(-\frac{(t-s)^{4}}{24}-\frac{(t-s)^{3}}{6} s\right. \\
& \left.+\frac{(t-s)^{2}}{2} s^{2}\right) \beta(s) \mathrm{d} s \\
& P_{32}(t)=\int_{0}^{t}\left(-\frac{(t-s)^{4}}{24} s-\frac{(t-s)^{3}}{6} s^{2}\right) y_{2}(s) \mathrm{d} s \\
& +\int_{0}^{t}\left(\frac{-(t-s)^{4}}{24}-\frac{(t-s)^{3}}{6} s+\frac{(t-s)^{2}}{2} s^{2}\right) y_{1}(s) \mathrm{d} s \\
& +\int_{0}^{t}\left(\frac{3(t-s)^{2}}{2} s-(t-s) s^{2}\right) y_{2}(s) \mathrm{d} s \\
& +\int_{0}^{t}\left(\frac{3(t-s)^{2}}{2}-5(t-s) s+s^{2}\right) y_{1}(s) \mathrm{d} s \\
& P_{33}(t)=-\frac{t^{4}}{24} \\
& P_{41}(t)=\int_{0}^{t} \frac{(t-s)^{2}}{2} s y_{2}(s) \mathrm{d} s \\
& +\int_{0}^{t}\left(\frac{(t-s)^{2}}{2}-(t-s) s\right) y_{1}(s) \mathrm{d} s-\frac{t^{4}}{24} y_{2}(0) \\
& P_{42}(t)=\int_{0}^{t}\left(\frac{(t-s)^{4}}{24}+\frac{(t-s)^{3}}{6} s\right) y_{2}(s) \mathrm{d} s \\
& +\int_{0}^{t}-\frac{(t-s)^{2}}{2} s y_{1}(s) \mathrm{d} s+\frac{t^{4}}{24} y_{1}(0) \\
& -\frac{t^{4}}{24} y_{2}(0) \\
& Q_{4}(t)=-h T\left(r_{\mathrm{ci}}-r_{\mathrm{gy}}\right) \int_{0}^{t}-\frac{(t-s)^{2}}{2} s \beta(s) \mathrm{d} s \\
& +\int_{0}^{t}\left(-\frac{(t-s)^{2}}{2}+(t-s) s\right) y_{2}(s) \mathrm{d} s \\
& +\int_{0}^{t}(2(t-s)-s) y_{2}(s) \mathrm{d} s \\
& -\frac{t^{2}}{2} y_{1}(0)-K \frac{t^{4}}{24} \beta(0) \\
& P_{44}(t)=-\frac{t^{4}}{24} \\
& Q_{1}(t)=h T\left(r_{\mathrm{ci}}-r_{\mathrm{gy}}\right) \int_{0}^{t}\left(-\frac{(t-s)^{3}}{6} s+\frac{(t-s)^{2}}{2} s^{2}\right) \beta(s) \mathrm{d} s \\
& +\int_{0}^{t}\left(\frac{(t-s)^{3}}{3}-2(t-s)^{2} s+(t-s) s^{2}\right) y_{2}(s) \mathrm{d} s \\
& -\int_{0}^{t}\left(3(t-s)^{2}-6(t-s) s+s^{2}\right) y_{1}(s) \mathrm{d} s+\frac{t^{3}}{3} y_{1}(0) \\
& Q_{2}(t)=h T\left(r_{\mathrm{ci}}-r_{\mathrm{gy}}\right) \int_{0}^{t}\left(\frac{(t-s)^{3}}{2} s^{2}-\frac{(t-s)^{2}}{2} s^{3}\right) \beta(s) \mathrm{d} s \\
& +\int_{0}^{t}\left(-(t-s)^{3} s+3(t-s)^{2} s^{2}-(t-s) s^{3}\right) y_{2}(s) \mathrm{d} s \\
& +\int_{0}^{t}\left(-(t-s)^{3}+9(t-s)^{2} s-9(t-s) s^{2}+s^{3}\right) \\
& \times y_{1}(s) \mathrm{d} s
\end{aligned}
$$

